\title{
Avaliação da assistência à saúde da mulher e da criança em localidade urbana da região Sudes- te do Brasil
}

Evaluating mother-and-child health care in Brazil

Sílvia R Santos, Antonio José LA Cunha, Cristiano M Gamba, Fabrício G Machado, Joaquim Maurício M Leal Filho e Nelson Luís M Moreira

Fundação Educacional Serra dos Órgãos. Teresópolis, RJ, Brasil 


\title{
Avaliação da assistência à saúde da mulher e da criança em localidade urbana da região Sudeste do Brasil* Evaluating mother-and-child health care in Brazil
}

\author{
Sílvia R Santos, Antonio José LA Cunha, Cristiano M Gamba, Fabrício G Machado, \\ Joaquim Maurício M Leal Filho e Nelson Luís M Moreira
}

Fundação Educacional Serra dos Ó rgãos. Teresópolis, RJ, Brasil

\section{Descritores}

Saúde materno-infantil”. Cuidados primários de saúde". Prestação de cuidados de saúde ${ }^{\#}$. Serviços de saúde infantil, utilização\#. Serviços de saúde materna, utilizaçãō". Diagnóstico da situação em saúde. Promoção da saúde. Vacinação.

\section{Keywords}

Maternal and child health". Primary health care $e^{\#}$. Delivery of health care ${ }^{\#}$. Child health services, utilization". Maternal health services, utilization ${ }^{\#}$. Diagnosis of health situation. Health promotion. Vaccination.

\section{Resumo}

Objetivo

Analisar e comparar os cuidados primários prestados à população materno-infantil e contribuir para a avaliação da assistência integral a esse grupo.

\section{Métodos}

Inquérito populacional realizado por entrevistas, no principal posto de vacinação do Município de Teresópolis, RJ, no Dia Nacional de Vacinação, que abrangeu questões sobre utilização de serviços de saúde e prestação de cuidados primários preventivos. Resultados

Foram colhidas informações de 329 crianças e suas respectivas mães. Mais de $90 \%$ das crianças haviam comparecido à consulta pediátrica nos três meses anteriores e quase todas possuíam o cartão da criança, embora em 30\% desses cartões não havia qualquer peso registrado no período. Observou-se associação positiva entre consulta de puericultura e registro de peso no cartão da criança ( $\mathrm{RP}=1,34$; IC: $1,13-1,58 ; p=0,0002)$. Cerca de $59 \%$ das mães compareceram à consulta de revisão de parto, mas $25 \%$ referiram nunca ter feito exame colpocitológico-oncótico e $36 \%$ nunca haviam realizado exame de mama. Observou-se associação positiva entre a idade materna acima de 20 anos e a realização de algum exame colpocitológico-oncótico durante a vida reprodutiva $(\mathrm{RP}=1,56$; IC: $1,08-2,26 ; p=0,03)$. Quase $70 \%$ das mães relataram uso de algum método anticoncepcional, principalmente pílula, condom e laqueadura tubária.

Conclusões

Apesar de algumas limitações, os resultados sugerem a viabilidade da metodologia utilizada, permitindo a identificação de deficiências importantes na prestação de cuidados primários de saúde para crianças e principalmente para mães.

\section{Abstract}

\section{Objective}

To describe and analyze primary health care provided to mother-and-child population in Teresópolis, state of Rio de Janeiro, Brazil.

Methods

A population survey was performed at the main vaccination station on the local Vaccine National Day, to collect information about the use of health care services and preventive primary care. 


\begin{abstract}
Results
Information was collected from 329 mothers and their children. More than $90 \%$ of children had at least one pediatric visit in the past three months. Almost all had their growth chart, but in 30\% of them the child's weight was not registered. There was a positive association between having a routine visit and the registration of the child's weight in the card $(R P=1.34 ; I C: 1.13-1.58 ; \mathrm{p}=0.0002)$. Around $59 \%$ of the mothers had a medical visit after giving after birth; $25 \%$ said they had never had a Pap smear test before, and 36\% never had a breast examination. There was a positive association between the mother's age being over 20 years and ever having a Pap smear test $(R P=1.56 ; I C: 1.08-2.26 ; \mathrm{p}=0.03)$. Almost $70 \%$ of the mothers referred the use of any contraceptional method, and the most common used were contraceptive pills, condoms and tubal ligation.
\end{abstract}

Conclusions

Despite some limitations, the results suggest a valid and useful methodology, which allows the identification of important needs in primary health care delivery to children and their mothers.

\section{INTRODUÇÃO}

No Brasil, ao longo da década de 80, o Ministério da Saúde e as Secretarias de Saúde estaduais e municipais, com o apoio de entidades internacionais e filantrópicas, implementaram programas voltados aos problemas mais prevalentes e de alta morbidade e mortalidade, com ênfase em ações de tecnologia de baixo custo e fácil acesso, tais como os Programas de Assistência Integral à Saúde da Mulher [PAISM] e da Criança [PAISC]. ${ }^{7}$ Nesse mesmo período, iniciou-se processo de reforma do setor saúde, com a aprovação da nova Carta Constitucional de 1988 e o estabelecimento do Sistema Único de Saúde (SUS). A universalidade e a eqüidade de acesso, a descentralização da gestão administrativa e a integração dos serviços de saúde passaram a ser metas políticas a serem alcançadas.

Muitas têm sido as resistências à consolidação da reforma sanitária brasileira. Com o agravamento da crise financeira no País, os problemas se agigantam e o sistema de saúde se apresenta com carência de recursos e organização deficiente. Por outro lado, são poucos os estudos de avaliação conjunta dos programas já implementados. ${ }^{8} 15$ As investigações realizadas têm se concentrado sobretudo na avaliação das ações preconizadas em cada programa, como estudos de frequiência de aleitamento materno, ${ }^{3}$ diarréia aguda,${ }^{2}$ infecções respiratórias agudas ${ }^{1}$ e cobertura vacinal, ${ }^{13}$ ou têm analisado o impacto isolado de cada programa. ${ }^{5,6,9,10}$

Nos últimos anos, a Organização Mundial de Saúde (OMS) e a Organização Pan-Americana de Saúde (OPAS) vêm desenvolvendo e propondo uma nova iniciativa voltada para a redução da mortalidade e para a melhora da saúde de crianças em países em desenvolvimento. A estratégia do AIDPI (Atenção Integrada às Doenças Prevalentes da Infância) ${ }^{11}$ tem como prioridade a melhoria da qualidade da assistência à criança doente, e o Ministério da Saúde do Brasil vem se empenhando na implementação dessa iniciativa. Nesses momentos de dificuldades econômicas e de mudanças estruturais, um melhor conhecimento a respeito da qualidade da assistência prestada à população maternoinfantil, em nível de atenção primária, torna-se fundamental para a reorientação de políticas de saúde e implementação de novas estratégias, assim como para a alocação de recursos.

Tradicionalmente, estudos de avaliação da assistência à saúde têm sido realizados por meio de inquéritos populacionais, ${ }^{14}$ nos quais uma amostra representativa da população é estudada, geralmente em seu domicílio. Face às limitações cada vez maiores de recursos, torna-se necessária a busca de alternativas metodológicas, como a realização de entrevistas em dias nacionais de vacinação, metodologia que já se mostrou viável em estudos anteriores. ${ }^{3}$

Assim, pois, pela utilização de uma metodologia simplificada e a partir de indicadores do processo de assistência, ${ }^{4}$ o presente estudo tem por objetivo descrever, analisar e comparar os cuidados primários prestados a crianças menores de um ano e suas mães, no Município de Teresópolis, RJ, Brasil, contribuindo para a avaliação da assistência integral à saúde prestada a esse grupo populacional e para o planejamento de novas ações de saúde.

\section{MÉTODOS}

O Município de Teresópolis localiza-se na Região Serrana do Estado do Rio de Janeiro, a 90 km da capital, com uma extensão de $849 \mathrm{~km}^{2}$. Sua população, estimada para 1998 em cerca de 127.000 habitantes, distribui-se prioritariamente em área urbana (84\%) e cerca de $16 \%$ dos teresopolitanos vivem em áreas carentes (favelas). As principais atividades econômicas da região são a agricultura e o turismo. 
Além da rede privada, a cidade dispõe de uma rede pública (própria e conveniada ao Sistema Único de Saúde) de serviços de atenção materno-infantil composta por dez unidades primárias, seis em área rural e quatro em área urbana, e três unidades secundárias, além de serviço de emergência em hospital universitário. No período de 1990 a 1995, o coeficiente de mortalidade infantil no Município de Teresópolis variou de 28,92 a 22,08 (por 1.000 nascidos vivos) e, em 1995, o coeficiente de mortalidade materna era de 35,05 (por 100.000 nascidos vivos), valores esses inferiores às médias do Estado do Rio de Janeiro. Em 1996, a cobertura pré-natal (seis ou mais consultas) foi de cerca de $70,5 \% .^{12}$

Os Dias Nacionais de Vacinação Antipólio foram instituídos em todo o Brasil em 1980. Além do esquema básico de vacinas nesses últimos quase 20 anos, a vacinação indiscriminada da população de zero a quatro anos completos tem sido realizada em um só dia, duas vezes ao ano. A partir de 1990, a multivacinação foi instituída, com a administração também de vacinas injetáveis. Recentemente, os Dias Nacionais de Vacinação têm sido também utilizados para a implementação de outras metas do Ministério da Saúde, como a busca de casos de hanseníase e distribuição de vitamina A. Em Teresópolis, nos últimos anos, a cobertura vacinal antipólio nos Dias Nacionais de Vacinação atinge $100 \%$ da populaçãoalvo. ${ }^{12}$ Dos 50 postos de vacina (PV) existentes no município, o PV n. ${ }^{\circ} 1$ é o de maior movimento, o melhor equipado e de localização mais central. Na primeira etapa da Campanha de Multivacinação do ano de 1998 , cerca de $16 \%$ de todas as crianças menores de um ano vacinadas em Teresópolis foram atendidas no PV ${ }^{\circ} 1$.

Para descrever, analisar e comparar os cuidados primários prestados à população materno-infantil em Teresópolis, foram entrevistadas todas as mães ou responsáveis por crianças menores de um ano de idade que compareceram ao PV n ${ }^{\circ} 1$ em 15 de agosto de 1998, Dia Nacional de Vacinação (segunda dose).

As entrevistas foram realizadas por meio de questionário pré-testado, com perguntas fechadas e abertas. Os entrevistadores foram estudantes de medicina $\left(5^{\circ}\right.$ e $6^{\circ}$ períodos $)$ e de enfermagem $\left(7^{\circ}\right.$ e $8^{\circ}$ períodos), devidamente treinados para a abordagem das mães quando da saída do posto de vacinação. As entrevistas foram realizadas com as mães confortavelmente sentadas. A maior parte das questões referiram-se a fatos recentes ocorridos na vida da entrevistada e da criança. Todo o processo de coleta de dados foi feito sob a supervisão de um dos coordenadores do estudo.
Foram coletadas as seguintes informações em relação à criança: (1) local de moradia, (2) idade, (3) sexo, (4) realização de consulta com médico pediatra nos últimos três meses, motivo alegado e natureza do serviço de saúde (atenção primária/emergência, público/ privado), (5) apresentação do Cartão da Criança, (6) registro de algum peso na curva ponderal nos últimos três meses e posição do peso na curva, (7) situação vacinal e identificação de eventual vacina em atraso.

Em relação à mãe, foram coletados os seguintes dados: (1) local de moradia, (2) idade, (3) comparecimento à consulta de revisão de parto, (4) realização de exame colpocitológico-oncótico e exame de mama, (5) uso atual de método contraceptivo e identificação do método, (6) percepção de estado atual de saúde e (7) identificação de eventual problema de saúde.

Em caráter exploratório, foram estudadas possíveis associações entre as seguintes variáveis em relação à criança: variáveis independentes: (1) idade materna (mãe adolescente), (2) local de moradia (favela), (3) motivo da consulta pediátrica (puericultura) e (4) natureza do serviço de saúde (público). Variáveis dependentes: (1) existência de algum registro de peso na curva ponderal nos últimos três meses, (2) posição do peso na curva e (3) vacinação em dia.

Em relação à mãe da criança: variáveis independentes: (1) idade materna (adolescente) e (2) local de moradia (favela). Variáveis dependentes: (1) comparecimento à consulta de revisão do parto, (2) realização de exame colpocitológico-oncótico e exame de mama.

Utilizou-se o Epi Info 6.04b para o armazenamento, a verificação da consistência dos dados e a análise estatística. Para a análise de associações, utilizou-se a razão de prevalência (RP) e para a inferência estatística, o teste do qui-quadrado, intervalo de confiança (IC) com o limite de significância estatística de 0,05.

\section{RESULTADOS}

Do universo de menores de um ano vacinados (segunda dose), $366(15,8 \%)$ o foram no PV $\mathrm{n}^{\circ} 1$. Desse total foram obtidas informações de 329 , ocorrendo $10,1 \%$ de perdas por recusa dos responsáveis em participar do estudo ou por incapacidade de abordagem de todas as díades - responsável-criança - em momentos de intenso movimento.

A quase totalidade das mães/crianças entrevistadas morava no Município de Teresópolis $(98,2 \%)$, sendo que $91 \%$ em área urbana. Somente 25 díades moradoras de Teresópolis $(7,8 \%)$ eram provenientes de bairros reconhecidamente carentes (favelas). 
Não foi observada diferença quanto à distribuição por sexo (49,5\% do sexo feminino) e a idade média das crianças era de 6 meses $(\mathrm{DP}= \pm 3,4 ;$ mediana $=6,0 ;$ moda $=11)$.

A grande maioria das crianças (93,3\%) foi consultada por médico pediatra pelo menos uma vez no trimestre anterior à entrevista. Nessa consulta, 55\% das crianças foram atendidas na rede pública de atenção primária, $42,3 \%$ na rede privada e $1,6 \%$ em serviço de emergência.

Os principais motivos para a consulta médica, tanto da rede pública quanto da rede privada, foram puericultura/consulta de rotina (66\%) e as infecções respiratórias agudas $(26,8 \%)$. Dentre as crianças atendidas em serviço de emergência, as infecções respiratórias agudas (IRA) foram o principal motivo da consulta (80\%).

A imensa maioria das mães ou responsáveis alegava possuir o Cartão da Criança (99,7\%), mas 6,7\% delas não o haviam trazido naquela ocasião. Daquelas em posse do cartão no momento da entrevista, $70,4 \%$ tinham pelo menos um peso assinalado no gráfico de crescimento (peso/idade) nos últimos três meses.

Dentre as 299 crianças consultadas por médico pediatra em ambulatório (público ou particular), nos três meses anteriores à entrevista, 25,4\% não tinham qualquer anotação relativa ao seu peso no cartão da criança. Analisando-se somente as crianças cujo motivo da consulta era puericultura $(n=202)$, observa-se que o peso não foi assinalado no cartão em $21,7 \%$ dos casos. Nas consultas por IRA, diarréia ou por outros motivos $(n=104)$ esse percentual era ainda maior $(31,7 \%)$.

Observou-se associação positiva de pequena magnitude entre consulta de puericultura e registro de peso no cartão da criança. $(\mathrm{RP}=1,34 ; \mathrm{IC}: 1,13-1,58 ; p=0,0002)$.

Não foram observadas associações estatisticamente significativas entre existir algum peso registrado na curva peso/idade do Cartão da Criança nos últimos três meses e o fato da mãe ser adolescente $(\mathrm{RP}=0,91$; IC: $0,73-1,12 ; p=0,33$, ou o bairro de moradia ser uma favela ( $\mathrm{RP}=0,82$; IC: $0,68-0,99 ; p=0,12)$, ou a consulta ter sido realizada em serviço público $(\mathrm{RP}=1,01$; IC: $0,88-1,17 ; p=0,84)$;

Das 216 crianças com pelo menos um peso assinalado no gráfico nos últimos três meses, $83,3 \%$ tinham esse peso situado entre as duas curvas (entre os percentis 10 e 90 ou entre os percentis 3 e 97 , dependendo do tipo de cartão), 12,5\% estavam com seu peso acima da curva superior e $4,2 \%$ estavam com seu peso abaixo da curva inferior. Das nove crianças com peso abaixo da curva inferior, uma estava no segundo mês de vida, enquanto todas as restantes tinham seis ou mais meses de idade. Das 27 crianças com peso acima do limite superior, $62,9 \%$ tinham seis ou mais meses de idade.

Não foram observadas associações estatisticamente significativas entre a criança ter seu peso situado entre as duas curvas e (1) o fato de a mãe ser adolescente (RP = 0,97; IC: 0,43-2,16; $p=0,93)$, (2) o bairro de moradia ser uma favela $(\mathrm{RP}=0,95$; IC: $0,86-1,04 ; p=$ $0,36)$ e (3) a consulta ter sido de puericultura ( $\mathrm{RP}=0,94$; IC: $0,94-1,06 ; p=0,97)$ ou ter sido realizada em serviço público ( $\mathrm{RP}=0,97$; IC: 0,91-1,03; $p=0,31)$.

Ao sair do PV, 93,6\% das crianças encontravam-se com sua vacinação básica em dia. As vacinas que mais estavam atrasadas eram DPT e BCG-ID.

Não foram observadas associações estatisticamente significativas entre a vacinação estar em dia e (1) o fato da mãe ser adolescente ( $\mathrm{RP}=1,04$; IC: 0,97-1,10; $p=0,37)$, (2) o bairro de moradia ser uma favela ( $\mathrm{RP}=0,97$; IC: 0,89 $1,06 ; p=0,60),(3)$ a consulta ter sido de puericultura $(\mathrm{RP}=1,00$; IC: $0,94-1,06 ; p=0,97)$ ou ter sido realizada em serviço público ( $\mathrm{RP}=0,97$; IC: 0,91-1,03; $p=0,31$ ).

Em 93,3\% das entrevistas realizadas, a mãe da criança estava presente e respondeu às perguntas específicas aos cuidados à saúde da mulher $(n=307)$.

A média da idade das mães foi 25,6 anos (DP $= \pm 6,2$ -mediana $=25,0 ;$ moda $=21,0$ ). Cinqüenta e quatro crianças (17,9\%) eram filhas de adolescentes (menores de 20 anos), sendo que três crianças tinham mães com idade abaixo de 15 anos $(0,9 \%)$. Nove crianças tinham mães com idade igual ou maior que 40 anos $(2,7 \%)$.

Somente $58,7 \%$ das mães haviam comparecido à consulta de revisão de parto dessa criança. Não foram observadas associações estatisticamente significativas entre o comparecimento à consulta de revisão de parto e o fato de a mãe ser adolescente $(\mathrm{RP}=0,96$; IC: $0,74-1,24 ; p=0,73$ ) ou o bairro de moradia ser uma favela (RP=1,25; IC: 0,79-1,98; $p=0,28)$.

Quanto à prevenção de câncer de colo de útero, 25,3\% das 304 mães relataram nunca ter realizado exame preventivo de câncer de colo de útero. Dentre as 227 mães que alguma vez realizaram exame colpocitológico-oncótico, em 17,6\% o último exame foi durante o pré-natal, $38,8 \%$ na consulta de revisão pós-parto e $43,6 \%$ em período de um a dez anos anteriores à gravidez.

Em relação ao exame das mamas, $35,7 \%$ das 305 mães referiram nunca ter sido submetidas a exame de 
mama. Dentre as 196 mães que alguma vez tiveram suas mamas examinadas, em $25 \%$ o último exame foi durante o pré-natal, $56,6 \%$ na consulta de revisão pós-parto e $18,4 \%$ em período de um a dez anos anteriores à gravidez.

Observou-se associação positiva de pequena magnitude entre a idade materna acima de 20 anos e a realização de algum exame colpocitológico-oncótico durante a vida reprodutiva $(\mathrm{RP}=1,56$; IC: $1,08-2,26 ; p=0,03)$. Foi observada tendência a associação positiva de pequena magnitude quanto à realização de algum exame de mama ( $\mathrm{RP}=1,42$; IC: $1,01-1,98 ; p=0,06)$ e a idade materna acima de 20 anos.

No que diz respeito à moradia em áreas carentes, não foram observadas associações estatisticamente significativas em relação à realização de algum exame colpocitológico-oncótico ( $\mathrm{RP}=1,02$; IC:0,64-1,62; $p=$ $0,94$ ) ou de mama (RP=1,24; IC:0,82-1,89; $p=0,24)$.

A maior parte das mães $(68,6 \%)$ referiu estar em uso de algum método anticoncepcional. Os métodos reversíveis foram os mais citados, seguidos pela laqueadura tubária. A idade média das mães laqueadas foi 31,4 anos $(\mathrm{DP}= \pm 4,6$; mediana $=31,0$; moda $=$ $31,0)$. Dentre as mães que haviam comparecido à consulta de revisão de parto $(n=178), 21,6 \%$ não estavam em uso de anticoncepção.

Somente $8,9 \%$ das mães referiram ter algum problema de saúde, sendo a hipertensão arterial o mais citado (em $36,7 \%$ dos casos).

\section{DISCUSSÃO}

Os resultados da presente pesquisa devem ser interpretados com cautela. O estudo limitou-se a colher informações de mães e de crianças, usuárias de um único posto de vacinação no Município de Teresópolis. Embora a distribuição das mães por faixa etária na amostra estudada (17,9\% de mães adolescentes) aproxime-se muito da distribuição na população brasileira, e sendo esse o principal posto de vacinação do município (maior, mais central e de mais fácil acesso), não se pode afirmar que a amostra estudada represente a população do município. No entanto, uma vez que a prevalência de moradores oriundos de áreas carentes $(7,8 \%)$ é aproximadamente a metade da prevalência da população no Município de Teresópolis (16\%), pode-se presumir que os resultados da presente pesquisa descrevem uma situação mais favorável do que a situação real do atendimento no município como um todo.

Outra limitação diz respeito à validade de alguns dados colhidos por entrevistas, particularmente aque- les que dependem da memória e da boa vontade da informante. Os possíveis aspectos de aferição podem ter sido minimizados pelo fato das entrevistas terem sido realizadas com as mães sentadas, e as perguntas limitadas, na sua maior parte, a fatos recentes.

Apesar das limitações, os resultados sugerem a viabilidade da metodologia utilizada. Além de revelarem informações anteriormente desconhecidas, permitem a identificação de deficiências importantes no processo de prestação de cuidados primários de saúde para a população infantil e sobretudo para a população de mães e mulheres de Teresópolis.

A grande maioria das crianças possui o cartão da criança, mas a monitorização do crescimento infantil parece ainda não receber a devida atenção, uma vez que cerca de $30 \%$ das crianças que tiveram contato com pediatra não tiveram seu peso registrado no cartão de crescimento, mesmo quando a consulta era de puericultura. Nesse caso, entretanto, o pediatra parece estar mais alerta à necessidade do registro de peso da criança no cartão do que em consultas motivadas por queixa específica. Cerca de $7 \%$ das crianças saíram do posto de multivacinação com seu calendário vacinal básico ainda atrasado. Esse dado possivelmente reflete os problemas de atraso na distribuição de algumas vacinas, particularmente DPT, que ocorreram em diversos municípios brasileiros no final de 1997 e início de 1998.

Dentre as diversas ações dos programas de atenção à saúde da mulher, destacam-se, negativamente, a baixa cobertura da consulta de revisão de parto, muito menor que a cobertura de pré-natal observada no município (70,5\%); o percentual elevado de mães que, em oportunidades recentes, passaram pelo menos por um serviço de saúde e referiram nunca terem sido submetidas a exame colpocitológico ou exame de mama. Uma vez que exames de mama durante o pré-natal e durante a internação do parto são mandatários, não somente para a saúde da mulher, como para a de seu filho, no que se refere ao incentivo ao aleitamento materno, a sua não realização indica sérios problemas na qualidade da atenção. Aparentemente, a mãe adolescente estaria menos sujeita à realização de exame colpocitológico-oncótico do que as mães com idade acima de 20 anos. No entanto, esse achado pode refletir somente menor oportunidade de ter utilizado algum serviço de saúde, em função do menor tempo de idade reprodutiva.

No último trimestre de 1998, o Ministério da Saúde realizou ampla campanha nacional de prevenção de câncer ginecológico. Desta forma, estudos subseqüentes seriam oportunos para investigar o im- 
pacto desta iniciativa na saúde das mulheres de Teresópolis.

Mais de 20\% das mães que tiveram contato recente com seu médico em consulta de revisão de parto não faziam uso de qualquer método contraceptivo e não praticavam abstinência sexual. As razões alegadas para este fato não foram exploradas no presente estudo, se era uma opção consciente ou falta de orientação da equipe de saúde.

Os indicadores de saúde, em especial os coeficientes de mortalidade infantil e materna do Município de Teresópolis estão bem melhores do que a média estadual e nacional. ${ }^{12}$ No entanto, os resultados do presente estudo sugerem que o processo de atendimento apresenta diversos problemas, especialmente no que diz respeito às ações básicas de promoção à saúde e prevenção de doenças, implementadas há mais de uma década, comprometendo a qualidade da atenção. Esses problemas dizem mais respeito ao compor-

\section{REFERÊNCIAS}

1. Amaral JJF, Menezes AMB, Halpern R, Victora CG, Barros FC. Prevalência e fatores de risco para infecção respiratória aguda em crianças aos seis meses de vida em Pelotas, RS. In: Benguigui $Y$, editor. Investigações operacionais sobre o controle das infecções respiratórias agudas (IRA). Washington (DC): OPAS; 1997. p. 85-100.

2. Campos GJ, Reis Filho SA, Silva AAM, Novochadlo MAS, Silva RA, Galvão CE. Morbimortalidade infantil por diarréia aguda em área metropolitana da região Nordeste do Brasil, 1986/1989. Rev Saúde Pública 1995;29:132-9.

3. Carvalhaes MABL, Parada CMGL, Manoel CM, Venâncio SY. Diagnóstico da situação do aleitamento materno em área urbana do Sudoeste do Brasil: utilização de metodologia simplificada. Rev Saúde Pública 1998;32:430-6.

4. Donabedian A. The quality of medical care. Science 1978;200:856-64

5. Favareto J, Thompson Z. Avaliaçào do programa de estímulo ao aleitamento materno do Hospital Universitário Regional do Norte do Paraná-Londrina. J Pediatr 1999;67:388-92.

6. Leite ALA, Nunes VRR, Teixeira RMV, Souza CG, Itho SF, Weigert AM et al. Resultados da Unidade de Terapia de Reidratação Oral implantada em hospital infantil. J Pediatr 1990;66:251-6.

7. Ministério da Saúde. Assistência integral à saúde da criança: bases de ação programática. Brasília; 1984.

8. Moura EC. Avaliação de serviços de saúde: um desenho epidemiológico [dissertação]. Campinas (SP): Faculdade de Ciências Médicas da Unicamp; 1994. tamento da equipe de saúde, em situação de rotina, do que à carência de recursos, e têm sido enfrentados por meio de campanhas de ampla mobilização e curta duração, ou pela distribuição de manuais e outras atividades cognitivas de educação continuada.

Mais do que a barreira do conhecimento, as dificuldades para a implementação de novas ações de saúde parecem se concentrar na área da motivação e da mudança de comportamento e de atitudes da equipe de saúde. No momento, esse parece ainda ser o grande desafio a ser enfrentado por planejadores, administradores e educadores da saúde.

\section{AGRADECIMENTOS}

À Secretaria Municipal de Saúde de Teresópolis, aos Professores Alvimar Gonçalves Delgado, Daniel Pinheiro Hernandez e Alvaro Chrispino e à equipe docenteassistencial da Unidade Básica de Saúde da Beira Linha, que contribuíram para a realização do estudo.

9. Moura EC. The relationship between the use of primary health care and infant health status at 12 months in a Brazilian community. Rev Bras Epidemiol 1998;1:79-87.

10. Oliveira, MC. Avaliação do impacto das ações de controle das infecções respiratórias agudas em Pelotas, RS, Brasil: uma investigação operativa. In: Benguigui $Y$, editor. Investigações operacionais sobre o controle das infecções respiratórias agudas (IRA). Washington (DC): OPAS; 1997. p.177-83.

11. Organización Panamericana de la Salud. Atención integrada a las enfermidades prevalentes de la infancia. Washington (DC); 1996.

12. Secretaria de Estado de Saúde. Departamento de Dados Vitais. Dados vitais. Rio de Janeiro; 1997.

13. Valente JG. Cobertura de vacinação no Estado do Rio de Janeiro em 1984 [dissertação]. Rio de Janeiro (RJ): Escola Nacional de Saúde Pública; 1987.

14. Victora CG, Barros FC, Tomasi E, Ferreira FS, MacAuliffe J, Silva AC et al. A saúde das crianças dos estados do Ceará, Rio Grande do Norte e Sergipe, Brasil: descrição de uma metodologia para diagnósticos comunitários. Rev Saúde Pública $1991 ; 25: 218-25$.

15. Westphal MF, Ribeiro MLG, Bogus CM. Avaliação das ações básicas de saúde e educação realizadas pela CNBB: Pastoral da Criança em duas comunidades do Município de São Paulo, 1989. São Paulo: NUPENS; 1991 